\section{Genome update: sigma factors in 240 bacterial genomes}

\section{Genomes of the month}

Ten new microbial genomes were published since the last 'Genome Update' column was written. The collection of this month's prokaryotic genomes, listed in Table 1, consists of one archaeon (Sulfolobus acidocaldarius) and five bacteria (Corynebacterium jeikeium, Haemophilus influenzae, Pseudomonas fluorescens, Rickettsia felis and Xanthomonas campestris). In addition, four microbial eukaryotic genomes have been published: Dictyostelium discoideum AX4 (Eichinger et al., 2005), Theileria annulata (Gardner et al., 2005), Theileria parvarich (Pain et al., 2005) and Toxoplasma gondii (Khan et al., 2005).

Corynebacterium jeikeium is an opportunistic pathogen and causes systemic infections, particularly in immunocompromised patients/hosts. Broad-spectrum resistance to antimicrobial agents is a common feature of C. jeikeium clinical isolates. Tauch et al. (2005) have published the genome sequence of the clinical isolate C. jeikeium K411. This strain contains a circular chromosome of $\sim 2.5 \mathrm{Mbp}$ and a $\sim 15 \mathrm{kbp}$ bacteriocinproducing plasmid (pKW4). About half the C. jeikeium genes $(\sim 52 \%)$ constitute a 'chromosomal backbone' of conserved genes found in all four Corynebacteria species sequenced to date (C. glutamicum, C. efficiens, C. diphtheriae and C. jeikeium).

Haemophilus influenzae strain Rd was the first bacterium to have its genome completely sequenced (Fleischmann et al., 1995) and was also the first bacterial genome to be patented (O'Malley et al., 2005), lending to this genome sequence a certain historical significance. In the decade since 1995, about 250 bacterial genomes have been sequenced and for many bacterial species multiple genome sequences have become available (for example, note that for all six of the genomes listed in Table 1, at least one other genome has been sequenced from the same genus). A second $H$. influenzae isolate has now been sequenced (Harrison et al., 2005). It is not generally appreciated that the originally sequenced $H$. influenzae strain was a rough form of a serotype not normally associated with disease. This was not stated as such in the publication (Fleischmann et al., 1995) and in this light it is an improvement that the sequence of a pathogenic, non-typeable serotype of $H$. influenzae has now been completed.

The gene content of the newly sequenced H. influenzae strain $86-028 \mathrm{NP}$ was compared with the $H$. influenzae rough serotype d strain KW20 (Rd). In total, 280 ORFs were identified in strain $86-028 \mathrm{NP}$ that are absent in the previously sequenced $\mathrm{Rd}$ strain, and 169 of the genes found in the $\mathrm{Rd}$ strain were missing in strain 86-028NP. However, the Rd sequence had been annotated when genome sequences were largely terra incognita, and annotation by comparative genomic methods was not possible. Annotation techniques have improved substantially as hundreds of genomes have been sequenced and annotated. Several studies have indicated that bacterial genomes can be overannotated, and in the $H$. influenzae Rd genome there could be as many as 200 genes annotated that might not be real (Skovgaard et al., 2001). In addition to the 'extra' genes, there could well be missing genes, for example, any small non-coding RNAs that can play important regulatory roles. In our opinion, using the same set of 1709 genes annotated 10 years ago in the original $H$. influenzae GenBank file as 'gospel' is a missed chance to take advantage of the progress that has been made in 10 years of microbial genome annotation.

Of most interest are genes found in the new genome that are related to virulence. These include genes whose products are involved in adherence, of which 5 of 12 are present in strain Rd as well, and two others have contingency repeats (short stretches of simple base repeats, for example GGGGG, which can slip during replication, resulting in addition or deletion of a single base, changing the reading frame of the gene; Bayliss et al., 2001). A total of 52 genes were identified for LPS biosynthesis, only four of which are unique to $86-028 \mathrm{NP}$. At least eight LPS genes have contingency repeats. Iron acquisition and the oxidative stress response are important processes in determining virulence of $H$. influenzae. Strain 86-028NP has 21 genes involved in iron acquisition (of which 20 have homologues in $\mathrm{Rd}$ ) and the genes thought to be involved in the oxidative stress response are relatively conserved between the two species. While these simple comparisons suggest that the difference in virulence may lie in differences in adherence, this needs to be confirmed with experimental data.

Microbiology Comment provides a platform for readers of Microbiology to communicate their personal observations and opinions in a more informal way than through the submission of papers.

Most of us feel, from time to time, that other authors have not acknowledged the work of our own or other groups or have omitted to interpret important aspects of their own data. Perhaps we have observations that, although not sufficient to merit a full paper, add a further dimension to one published by others, or we may have a useful piece of methodology that we would like to share.

Guidelines on how to submit a Microbiology Comment article can be found in the Instructions for Authors at http://mic.sgmjournals.org

It should be noted that the Editors of Microbiology do not necessarily agree with the views expressed in Microbiology Comment.

Charles Dorman, Editor-in-Chief 
Table 1. Summary of the published genomes discussed in this update

Note that the accession number for each chromosome is the same for GenBank, EMBL and DDBJ.

\begin{tabular}{|c|c|c|c|c|c|c|c|}
\hline Name & Length & $\mathrm{A}+\mathrm{T}(\mathbf{m o l} \%)$ & No. of genes & tRNAs & rRNAs & $\sigma^{54} / \sigma^{70} / \mathrm{ECF} \sigma$ & Accession no. \\
\hline Corynebacterium jeikeium K411 (Main) & 2462499 & $38 \cdot 6$ & 2137 & 50 & 3 & $0 / 2 / 7$ & CR931997 \\
\hline Corynebacterium jeikeium K411 (pKW4) & 14323 & $46 \cdot 2$ & 16 & 0 & 0 & $0 / 0 / 0$ & AF401314 \\
\hline Haemophilus influenzae 86-028NP (Main) & 1913428 & $61 \cdot 8$ & 1792 & 58 & 6 & $0 / 2 / 2$ & СР000057 \\
\hline Pseudomonas fluorescens Pf-5 (Main) & 7074893 & $36 \cdot 7$ & 6137 & 71 & 5 & $1 / 4 / 28$ & СР000076 \\
\hline Rickettsia felis URRWXCal2 (Main) & 1485148 & $67 \cdot 5$ & 1400 & 33 & 1 & $0 / 2 / 0$ & CP000053 \\
\hline Rickettsia felis URRWXCal2 (pRF) & 62829 & $66 \cdot 4$ & 68 & 0 & 0 & $0 / 0 / 0$ & СР000054 \\
\hline Rickettsia felis URRWXCal2 (pRFdelta) & 39263 & $66 \cdot 8$ & 44 & 0 & 0 & $0 / 0 / 0$ & СР000055 \\
\hline Sulfolobus acidocaldarius DSM639 (Main) & 2225959 & $63 \cdot 2$ & 2223 & 48 & 1 & - & СР000077 \\
\hline Xanthomonas campestris 8004 (Main) & 5148708 & $35 \cdot 0$ & 4273 & 55 & 2 & $2 / 3 / 10$ & CР000050 \\
\hline
\end{tabular}

Pseudomonas fluorescens is a commensal plant bacterium that can produce antimicrobial compounds to suppress plant pathogens and may even function as a growth promoter for plants. As a biofilm producer it is implicated in the fouling of dairy products in particular. P. fluorescens Pf-5 is the fourth publicly available genome of the pseudomonads; it is the largest of the four pseudomonads sequenced so far and it is composed of a $7 \mathrm{Mb}$ circular chromosome, with 6144 annotated ORFs, $63 \%$ of which have been assigned a function and 330 of which have no significant similarity to known proteins (Paulsen et al., 2005). Other Pseudomonas genomes in public databases are from $P$. aeruginosa $\mathrm{PAO} 1, P$. putida KT2440 and $P$. syringae DC3000. Limited gene synteny exists between the genome of $P$. fluorescens Pf-5 and the other pseudomonads that have been sequenced. The authors suggest the existence of a core of over 4000 genes that are conserved between the four species. Features related to secondary metabolism have been localized to nine gene clusters. One such cluster encodes hydrogen cyanide production and is also found in

P. aeruginosa. The chromosome contains one phage- and seven prophage-related genomic islands, constituting roughly $268 \mathrm{kbp}$ in total (Paulsen et al., 2005). $P$. fluorescens Pf5 is the largest genome shown in Table 1, and it also has by far the largest number of sigma factors - a total of $33\left(1 \sigma^{54}, 4 \sigma^{70}\right.$ and 28 ECF $\sigma-$ see below for more details).

Rickettsia felis (Ogata et al., 2005) is the largest of the seven currently finished Rickettsia genomes. Described in 1990 as a Rickettsia-like micro-organism, this flea-borne bacterium was proposed in 1996 to be a distinct species, Rickettsia felis. Originally characterized as a typhus-like Rickettsia, phylogenetic analysis has reclassified $R$. felis into the spotted fever group of Rickettsiae. After its discovery in 1990 in fleas in the Americas, the first European human case of an R. felis infection was reported in August 2000 (Marquez et al., 2002).

As $R$. felis is an obligate intracellular parasite, phenotypic characterization was difficult before post-genomic studies. There are several nice examples where predictions made from the $R$. felis genome sequence could be verified experimentally (Ogata et al., 2005). For example, ORFs with close sequence similarity to bacterial pili-associated genes led to the electron microscopy discovery of both conjugationand attachment-associated forms of pili. Perhaps the most important finding in $R$. felis is the presence of plasmids carrying conjugation-associated genes. As the gene content of the plasmids can be changed, this could provide a new tool for studying the more pathogenic species of Rickettsia.

The model Crenarchaeota organism, Sulfolobus acidocaldarius strain DSM639, was the first hyperthermoacidophile to be characterized from terrestrial solfataras and is the third Sulfolobus genome to be sequenced (Chen et al., 2005). It has often been used in in vivo genetic studies of Archaea because of its ease of transformation and sensitivity to many ribosomal antibiotics. It grows optimally at $80^{\circ} \mathrm{C}$ and $\mathrm{pH} 2$ under aerobic conditions. The genome is $\mathrm{A}+\mathrm{T}$ rich (63 mol\%), $2 \cdot 2 \mathrm{Mbp}$ in length and carries 2292 predicted protein-encoding genes, of which more than $50 \%$ are specific to Sulfolobus. As is the case for S. solfataricus, there is evidence for three replication origins in S. acidocaldarius. Moreover, many single genes as well as the first genes in operons lack a well-defined Shine-Dalgarno motif. S. acidocaldarius differs from other known Sulfolobus species in that it accepts a more limited range of carbon sources for nutrition and contains genes for thermopsin, a UV damage excision repair system, an aromatic ring dioxygenase and a characteristic restriction modification system. It is worth noting that the integrated conjugative plasmid is likely to be involved in intercellular genetic exchange, and yet $S$. acidocaldarius appears to have a very stable genome organization.

Black rot is a disease of crucifers (cabbage family) caused by Xanthomonas campestris pv. campestris (Xcc), a species of Gram-negative, aerobic bacteria. Black rot symptoms initially appear as $\mathrm{V}$-shaped areas along the outer leaf edges; in the latter stages of the disease leaf veins turn black, plants become stunted, wilt and usually die. The genome of $X$. campestris strain 8004 was sequenced, annotated (Qian et al., 2005) and compared with that of $X$. campestris strain ATCC 33913 (da Silva et al., 2002). There is a large degree of sequence conservation between the two genomes, both at the amino acid level and at the nucleic acid level. Analyses of the genome sequence have identified already known virulence factors, several additional metabolic pathways and regulatory systems, such as those for fatty acid degradation, type IV secretion and cell signalling. The $X$. campestris strain 8004 genome contains two $\sigma^{54}$ genes, which normally exist in a 
single copy in most proteobacterial genomes. This will be discussed in more detail below.

\section{Method of the month - sigma factors in bacterial genomes}

Sigma factors allow sequence-specific binding of RNA polymerase to bacterial promoters. We have constructed three profile hidden Markov models (HMMs), to identify the genes for $\sigma^{54}, \sigma^{70}$ and ECF $\sigma$, based on experimentally verified sequences from the UNIPROT database (K. Kill, N. T. Hansen, L. J. Jensen, M. Skovgaard \& D. W. Ussery, unpublished). Using these we have looked at the distribution of the three classes of sigma factors throughout the 240 bacterial genomes currently in our database. As a general rule, it appears that the number and diversity of sigma factor genes per genome relates to the environmental variation allowing growth for a given species.

Notice that in the first panel of Fig. 1, most genomes have either one or no $\sigma^{54}$ genes, although a few of the Proteobacteria genomes can have two. So far, all sequenced Xanthomonas species have two $\sigma^{54}$ genes, as does Bordetella (with the exception of Bordetella pertussis). The future will tell whether the apparent trend in the presence of $\sigma^{54}$ genes according to phylum - either it is there in a single copy or it is absent - will continue to hold. Note that Fig. 1 illustrates the total number of chromosomes, not genomes. Thus, for example, in the
Spirochetes, the chromosomes with no $\sigma^{54}$ are actually secondary chromosomes, which means that all spirochaete genomes do indeed have one $\sigma^{54}$.

The $\sigma^{70}$ group is shown in the middle of Fig. 1; this group includes the household $\sigma$ factor, as well as $\sigma$ factors involved in the control of stationary phase, sporulation, flagella assembly and heat-shock response. There is a larger divergence in the numbers here than for $\sigma^{54}$, since each organism might only employ some of these mechanisms. For example, in the Actinobacteria, we see Streptomyces avermitilis and Streptomyces coelicolor with 13 and $14 \sigma^{70}$ genes, respectively, while the rest of the Actinobacteria have far less; but it is worth noting that the two Streptomyces genomes are about $9 \mathrm{Mbp}$, approximately three times the mean size of the other actinobacterial genomes. In the Bacteroidetes/Chlorobi group, we see that Chlorobium tepidum surprisingly lacks even an apparent household sigma factor. This is due to a frameshift in the gene, which according to the GenBank file is not a sequencing error. In the Cyanobacteria we find a quite high number of $\sigma^{70}$ genes, which in this case control functions that are very different to those in the other sequenced bacterial genomes. The household sigma factor in Deinococcus radiodurans $\mathrm{R} 1$ is so divergent from anything else, that it is not picked up by our HMM. The Firmicutes are interesting, because they contain a quite large range in the number of $\sigma^{70}$ genes. What is not seen in
Fig. 1 is that the Bacillus and Clostridium species have many $\sigma^{70}$ genes (6-8), while the remaining Firmicutes, such as staphylococci and streptococci have only one or two. The main difference seems to concern the presence or absence of sporulation factors. When looking at the Proteobacteria and the Spirochetes, we find only a low number of sigma factors. Although it seems that a number of these lack $\sigma^{70}$ completely, this is just an artifact of multiple chromosomes that is, all the Proteobacteria and Spirochetes have at least one $\sigma^{70}$ per genome.

ECF $\sigma$ factors are generally far more numerous than the other two classes, but since they are not essential, they are missing in many organisms. They are especially numerous in the Bacteroides and Streptomyces species, which have more than 40 ECF $\sigma$ factors each. Bacteroides thetaiotamicron currently holds the record with 48 predicted ECF $\sigma$ factors. In comparison, Chlorobium tepidum and Porphyromonas gingivalis, also of the Bacteroidetes/Chlorobi group, only have three and six, respectively. In the Firmicutes most genera do not have ECF $\sigma$ factors at all. This seems to correlate with the presence of multiple $\sigma^{70}$ factors, as it is only the Bacillus, Clostridium and Listeria species, plus Lactococcus lactis, that do have ECF $\sigma$ factors. In the Proteobacteria we see many genomes without ECFs, and most have less than five, although some, especially Xanthomonas and Pseudomonas species, have more. Actually Pseudomonas fluorescens PF-5 holds the record amongst
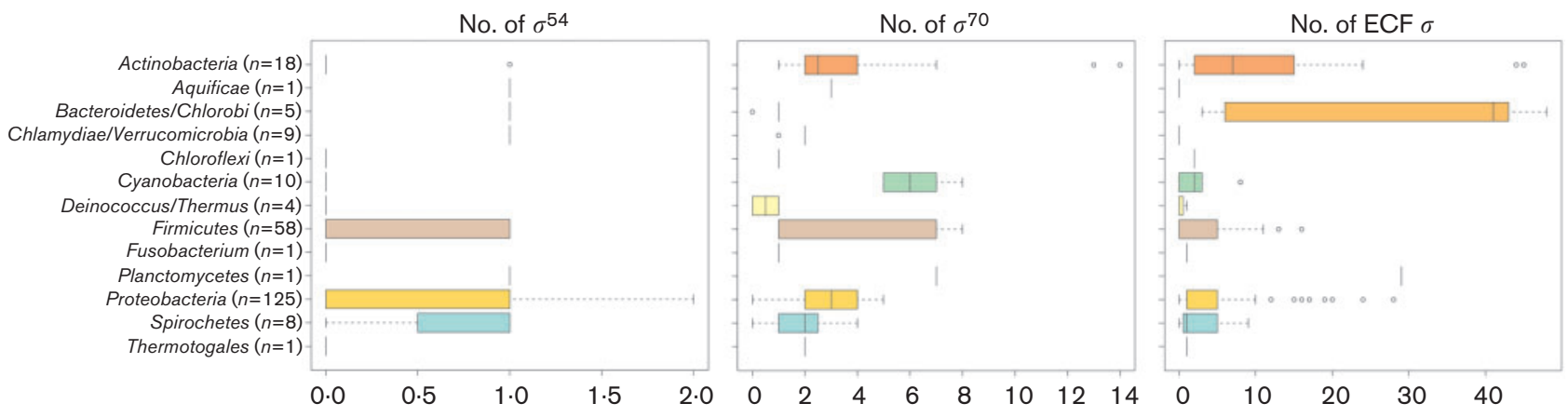

Fig. 1. Box and whisker plot of the number of sigma factor proteins in 13 different bacterial phyla. Note the difference in scales on the bottom axis. The colour scheme for the phyla is the same as found in the GenomeAtlas database (www.cbs.dtu.dk/services/GenomeAtlas/). The box represents the middle $50 \%$ of the data. The median of the data is shown by a vertical line. The 25th and 75th quartiles are shown on the left and right side of the median, respectively. The whiskers cannot extend any further than 1.5 times the length of the quartiles. Outlier data points outside the whiskers are shown by open circles. One single vertical line is shown where only one proteome is present. 
the Proteobacteria, with 28 predicted ECF $\sigma$ factors.

\section{Supplemental web pages}

Access to additional web pages containing supplemental material related to this article can be obtained via the following URL: http://www.cbs.dtu.dk/services/ GenomeAtlas/suppl/GenUp018/

\section{Acknowledgements}

This work was supported by a grant from the Danish Center for Scientific Computing.

\section{Kristoffer Kill, Tim T. Binnewies, Thomas Sicheritz-Pontén, Hanni Willenbrock, Peter F. Hallin, Trudy M. Wassenaar $†$ and David W. Ussery}

Center for Biological Sequence Analysis, BioCentrum-DTU, Building 208, The Technical University of Denmark, DK-2800 Kgs Lyngby, Denmark

†Present address: Molecular Microbiology and Genomics Consultants, Zotzenheim, Germany.

Correspondence: David W. Ussery (dave@cbs.dtu.dk)

Bayliss, C. D., Field, D. \& Moxon, E. R. (2001). The simple sequence contingency loci of Haemophilus influenzae and Neisseria meningitidis. J Clin Invest 107, 657-662.

Chen, L., Brugger, K., Skovgaard, M. \& 8 other authors (2005). The genome of Sulfolobus acidocaldarius, a model organism of the Crenarchaeota. J Bacteriol 187, 4992-4999.

da Silva, A. C., Ferro, J. A., Reinach, F. C. \& 59 other authors (2002). Comparison of the genomes of two Xanthomonas pathogens with differing host specificities. Nature 417, 459-463.

Eichinger, L., Pachebat, J. A., Glockner, G. \& 94 other authors (2005). The genome of the social amoeba Dictyostelium discoideum. Nature 435, 43-57.

Fleischmann, R. D., Adams, M. D., White, O. \& 37 other authors (1995). Whole-genome random sequencing and assembly of Haemophilus influenzae Rd. Science 269, 496-512.

Gardner, M. J., Bishop, R., Shah, T. \& 41 other authors (2005). Genome sequence of Theileria parva, a bovine pathogen that transforms lymphocytes. Science 309, 134-137.

Harrison, A., Dyer, D. W., Gillaspy, A. \& 10 other authors (2005). Genomic sequence of an otitis media isolate of nontypeable Haemophilus influenzae: comparative study with $H$. influenzae serotype d, strain KW20. J Bacteriol 187, 4627-4636.

Khan, A., Taylor, S., Su, C. \& 14 other authors (2005). Composite genome map and recombination parameters derived from three archetypal lineages of Toxoplasma gondii. Nucleic Acids Res 33, 2980-2992.

Marquez, F. J., Muniain, M. A., Perez, J. M. \& Pachon, J. (2002). Presence of Rickettsia felis in the cat flea from southwestern Europe. Emerg Infect Dis 8, 89-91.

Ogata, H., Renesto, P., Audic, S., Robert, C., Blanc, G., Fournier, P. E., Parinello, H., Claverie, J. M. \& Raoult, D. (2005). The genome sequence of Rickettsia felis identifies the first putative conjugative plasmid in an obligate intracellular parasite. PLoS Biol 3, e248.

O'Malley, M. A., Bostanci, A. \& Calvert, J. (2005). Whole-genome patenting. Nat Rev Genet 6, 502-506.

Pain, A., Renauld, H., Berriman, M. \& 47 other authors (2005). Genome of the host-cell transforming parasite Theileria annulata compared with T. parva. Science 309, 131-133.

Paulsen, I. T., Press, C. M., Ravel, J. \& 26 other authors (2005). Complete genome sequence of the plant commensal Pseudomonas fluorescens Pf-5. Nat Biotechnol 23, 873-878.

Qian, W., Jia, Y., Ren, S. X. \& 25 other authors (2005). Comparative and functional genomic analyses of the pathogenicity of phytopathogen Xanthomonas campestris pv. campestris. Genome Res 15, 757-767.

Skovgaard, M., Jensen, L. J., Brunak, S., Ussery, D. \& Krogh, A. (2001). On the total number of genes and their length distribution in complete microbial genomes. Trends Genet 17, 425-428.

Tauch, A., Kaiser, O., Hain, T. \& 13 other authors (2005). Complete genome sequence and analysis of the multiresistant nosocomial pathogen Corynebacterium jeikeium K411, a lipid-requiring bacterium of the human skin flora. J Bacteriol 187, 4671-4682.

\section{DOI 10.1099/mic.0.28339-0}

\section{Intragenic position of UUA codons in streptomycetes}

Streptomycetes have huge linear genomes ( $>8 \mathrm{Mbp}$ ), extreme GC content (around $70 \mathrm{~mol} \%$ ), and their life cycles involve vegetative growth, a phase with formation of aerial hyphae and a sporulation stage. In addition, they produce a range of secondary metabolites, including many of the antibiotics used in clinics today, and for this reason they have immense practical importance. Production of antibiotics is typically closely linked to differentiation. One of the key genes involved in the differentiation switch is bldA, which encodes the tRNA recognizing the very rare UUA (leucine) codons (Lawlor et al., 1987). It has been shown that the production of this tRNA is subject to temporal regulation as it becomes abundant in old cultures (Leskiw et al., 1993).

Conversely, genes containing UUA codons are often linked to antibiotic production or other aspects of differentiation (see reviews by Leskiw et al., 1991a; Chater, 1993). As a consequence, heterologous expression of proteins, which often takes place in liquid cultures under vegetative growth, can be problematic if the foreign gene contains UUA codons (Leskiw et al., 1991b; Ueda et al., 1993). Codon usage in the streptomycetes is therefore an interesting phenomenon that deserves full attention.

Wright \& Bibb (1992) investigated a limited number of streptomycete genes and concluded that codon usage largely reflected mutational bias. A full genomic analysis based on codon usage in Streptomyces coelicolor and Streptomyces avermitilis (both are fully sequenced and their genomes are available through GenBank) was recently published in Microbiology by Wu et al. (2005). The paper used a measure of synonymous codon usage bias called the codon adaptation index (Sharp \& Li, 1987) to predict highly expressed genes. Technically, this involves identification of genes using codons that are particularly abundant in highly expressed genes such as ribosomal genes. Thus, UUA codons and other single codons had no particular focus in that study. In fact, UUA has to my knowledge not received particular focus in bioinformatic studies of the streptomycete genomes so far. The aim of this Comment therefore is to apply a genomic view on UUA, thereby supplementing the work by $\mathrm{Wu}$ et al. and adding to the general knowledge about codon usage in these important bacteria.

I hypothesize that since transcripts for genes containing UUA codons are produced throughout the life cycle, initiation of translation on such mRNAs is futile, and on this account the fitness among different mutants in a population could depend on the loss of energy associated with this futile 